\title{
S? \\ Antiadiabatic limit of the exchange-correlation kernels of an inhomogeneous electron gas
}

\author{
V. U. Nazarov, ${ }^{1,2}$ I. V. Tokatly, ${ }^{3,4}$ S. Pittalis, ${ }^{5}$ and G. Vignale ${ }^{5}$ \\ ${ }^{1}$ Research Center for Applied Sciences, Academia Sinica, Taipei 115, Taiwan \\ ${ }^{2}$ Department of Physical Chemistry, Far-Eastern National Technical University, Vladivostok, Russia \\ ${ }^{3}$ Nano-Bio Spectroscopy group and ETSF Scientific Development Centre, Departamento de Física de Materiales, \\ Centro de Física de Materiales CSIC-UPV/EHU-MPC, Universidad del País Vasco, E-20018 San Sebastián, Spain \\ ${ }^{4}$ IKERBASQUE, Basque Foundation for Science, E-48011 Bilbao, Spain \\ ${ }^{5}$ Department of Physics, University of Missouri-Columbia, Columbia, Missouri 65211, USA \\ (Received 8 March 2010; revised manuscript received 8 May 2010; published 2 June 2010)
}

\begin{abstract}
We express the high-frequency (antiadiabatic) limit of the exchange-correlation (xc) kernels of an inhomogeneous electron gas in terms of the following equilibrium properties: the ground-state density, the xc kinetic stress tensor, the pair-correlation function and the ground-state xc potential. Of these quantities, the first three are amenable to exact evaluation by quantum Monte Carlo methods while the last can be obtained from the inversion of the Kohn-Sham equation for the ground-state orbitals. The exact scalar kernel, in this limit, is found to be of very long range in space, at variance with the kernel that is used in the standard local density approximation. The antiadiabatic xc kernels will be useful in calculations of excitation energies by timedependent density-functional theory in atoms, molecules, and solids, and provide a solid basis for interpolation between the low- and high-frequency limits of the xc kernels.
\end{abstract}

DOI: 10.1103/PhysRevB.81.245101

PACS number(s): 71.15.Mb, 31.15.ee, 71.45.Gm

\section{INTRODUCTION}

The exchange-correlation (xc) kernel is a quantity of central importance both in time-dependent density-functional theory (TDDFT) and in time-dependent current densityfunctional theory (TDCDFT). It is formally defined as the functional derivative of the time-dependent xc potential $V_{\mathrm{xc}}$ with respect to time-dependent density in TDDFT, or as the functional derivative of time-dependent xc vector potential $A_{\mathrm{xc}, i}$ with respect to time-dependent current-density $j_{j}$ in TDCDFT. In practice, this kernel connects the physical densityand current-response functions of the interacting many-body system to those of a fictitious noninteracting system-the so-called Kohn-Sham (KS) system-which has the exact ground-state density. More precisely, one has

$$
\chi^{-1}=\chi_{s}^{-1}-f_{\mathrm{xc}}-V_{C}\left(\left|\mathbf{r}-\mathbf{r}^{\prime}\right|\right)
$$

in TDDFT (Ref. 1) and

$$
\hat{\chi}^{-1}=\hat{\chi}_{s}^{-1}-\hat{f}_{\mathrm{xc}}-\frac{c}{e \omega^{2}} \partial V_{C}\left(\left|\mathbf{r}-\mathbf{r}^{\prime}\right|\right) \partial^{\prime}
$$

in TDCDFT. ${ }^{2}$ Here $\chi$ and $\hat{\chi}$ denote the exact density- and current-response functions (the second being a tensor, as emphasized by the hat), $\chi_{s}$ and $\hat{\chi}_{s}$ are their Kohn-Sham counterparts, $\omega$ is the frequency, $c$ is the velocity of light, $e$ is the absolute value of the charge of electron, and $V_{C}(r)=e^{2} / r$ is the Coulomb interaction.

The great importance of the $f_{\mathrm{xc}}$ kernels stems from the fact that the Kohn-Sham response functions usually misrepresent both the response and the excitation spectrum of the system. Poles of $\chi_{s}$, for example, occur at differences of Kohn-Sham eigenvalues, which are known to be, at least conceptually, unrelated to the true excitation energies of the system. It is the task of $f_{\mathrm{xc}}$ to shift the poles of the KS response functions from these unphysical values to the actual physical ones, and this is a very difficult task, particularly when it comes to band gaps and excitons.

Very few exact results are known about $f_{\mathrm{xc}}$. Even in the simplest case of a homogeneous electron gas (in which case $f_{\mathrm{xc}}$ and $\hat{f}_{\mathrm{xc}}$ are functions of $\left|\mathbf{r}-\mathbf{r}^{\prime}\right|$ and $\hat{f}_{\mathrm{xc}}$ decomposes into independent longitudinal and transversal components) very little is known. The static limit of $f_{\mathrm{xc}}(q, \omega)$ has been calculated by variational and diffusion quantum Monte Carlo (QMC) and fitted to simple formulas. ${ }^{3,4}$ It provides the basis for the so-called adiabatic approximation, which is based on the assumption that the exchange-correlation potential relaxes to the ground-state form on a time scale that is much shorter than the time scale of the evolution of the system. In the opposite limit of high frequency, the quantity $\lim _{\omega \rightarrow \infty} f_{\mathrm{xc}}(\omega) \equiv f_{\mathrm{xc}}^{\infty}$ is known from exact sum rules to be expressible in terms of the density, the ground-state kinetic energy, and the ground-state pair-correlation function (or equivalently the static structure factor), all of which can be obtained from QMC calculations. This limit provides the basis for an "antiadiabatic approximation," in which $f_{\mathrm{xc}}$ is interpreted as the stiffness of the ground-state wave function with respect to instantaneous virtual deformations. This point will be expounded more fully in the following.

The frequency dependence of the homogeneous kernels has been the object of several parametrizations, beginning with the one by Gross and Kohn in 1985 (Ref. 1) (see also Refs. 5 and 6), which interpolate between low- and highfrequency limits, and incorporate as many exact properties and constraints as possible. Perturbative calculations, which treat electron exchange exactly, have also been carried out. ${ }^{7,8}$

Historically, the xc kernels of the homogeneous electron gas have played (and still play) an important role as the basis for local density approximations (LDAs), whereby the xc kernels of the nonhomogeneous systems are approximated in terms of those of a homogeneous system, evaluated at the 
local density. However, it has become clear in recent years that some features of the exact kernels cannot be reproduced by the local density approximation. For example, it is now known that the exact scalar xc kernel has a singularity at finite frequency and $q \rightarrow 0$ (corresponding to long range in space), which plays an important role in the optical spectra of extended systems. ${ }^{9}$ Reining et al. ${ }^{10}$ introduced phenomenologically a singular $f_{\mathrm{xc}}$, and using it "on top" of bands calculated by the GW method demonstrated considerable improvement in the calculated optical spectrum of silicon. This singularity naturally appears in xc kernels derived from the many-body perturbation theory (see, e.g., Refs. 11-13). Another way to introduce a singular $f_{\mathrm{xc}}$ from first principles is to derive it from the tensor $\hat{f}_{\text {xc }}$ of TDCDFT, taken, e.g., within the LDA. Indeed, any regular approximation for $\hat{f}_{\mathrm{xc}}$, when translated into an approximation for $f_{\mathrm{xc}}$, does produce a singularity of the expected form. ${ }^{14,15}$ Promising results of the inclusion of the many-body interactions in the calculation of optical spectrum of a semiconductor have been achieved along these lines very recently. ${ }^{16}$ Further it now appears that not only the singular behavior of $f_{\mathrm{xc}}$ at $q=0$ but also the detailed nonlocal spatial dependence of this kernel plays a role in the calculation of the optical spectra.

In view of these developments it seems particularly timely to try and learn as much as possible about the exact properties of $f_{\mathrm{xc}}$ in nonuniform systems. In a recent paper we have taken a first step in this direction by deriving an exact expression for the $f_{\mathrm{xc}}$ of a weakly inhomogeneous system to second order in the strength of the external potential. ${ }^{17}$ This expression involves the kernels of the homogeneous electron gas (which, as discussed above, are still poorly described) but is otherwise fully nonlocal. Unfortunately, the perturbative approach restricts us to metals and seems to exclude $a$ priori the interesting case of semiconductors.

In this paper we calculate the exchange-correlation kernels for arbitrary nonhomogeneous systems in the antiadiabatic limit. As remarked above, the antiadiabatic limit of the homogeneous exchange-correlation kernel is well known (a state-of-the-art formula for it is provided in Appendix B). Here we extend that knowledge to the considerably more complex case of a nonhomogeneous system. In spite of the additional complication, we are still able to express the result in terms of ground-state density, kinetic exchange-correlation stress tensor, pair-correlation function, and exact exchangecorrelation potential. All these quantities are well within the reach of modern numerical algorithms and therefore our results open the way to an accurate study of $f_{\mathrm{xc}}$ in the antiadiabatic limit.

The high-frequency response of quantum many-body systems has recently come under intense scrutiny in the context of the continuum mechanics formulation of quantum manybody theory, ${ }^{18,19}$ which, in turn, can be considered as a particular realization of the time-dependent deformation functional theory (TDDefFT). ${ }^{20,21}$ In this approach one attempts to describe the dynamics in terms of a vector field $\mathbf{u}(\mathbf{r}, t)$, which corresponds to the displacement of an infinitesimal volume element from its "equilibrium position" in the ground state (in this theory the time evolution is viewed as a geometric deformation of the ground state). It turns out that the simplest approximation that leads to an eigenvalue problem for the excitation energies is in fact equivalent to a highfrequency expansion for the current response function. We get

$$
m \ddot{\mathbf{u}}(\mathbf{r}, t)=-\nabla V_{1}(\mathbf{r}, t)-\frac{1}{n_{0}(\mathbf{r})} \int d \mathbf{r}^{\prime} \mathbf{M}\left(\mathbf{r}, \mathbf{r}^{\prime}\right) \cdot \mathbf{u}\left(\mathbf{r}^{\prime}, t\right),
$$

where $n_{0}(\mathbf{r})$ is the ground-state density and the tensor $\mathbf{M}\left(\mathbf{r}, \mathbf{r}^{\prime}\right)$ is the first moment of the current excitation spectrum [see Eq. (5)]. This quantity can be expressed in terms of ground-state properties, i.e., it is the second variational derivative of the ground-state energy with respect to the instantaneous displacement field $\mathbf{u}(\mathbf{r})$, evaluated at $\mathbf{u}=0$. We will see that $\mathbf{M}\left(\mathbf{r}, \mathbf{r}^{\prime}\right)$ also plays a crucial role in the construction of $f_{\mathrm{xc}}^{\infty}$. In particular, we will demonstrate that $f_{\mathrm{xc}}^{\infty}$ can be derived directly from the quantum continuum mechanics, or, in other words, from the high-frequency limit of TDDefFT.

The main result of this work is the exact and explicit representation of the antiadiabatic limit $f_{\mathrm{xc}}^{\infty}\left(\mathbf{r}, \mathbf{r}^{\prime}\right)$ of the $\mathrm{xc}$ kernel in terms of a few characteristics of the ground state. Hence we add one more item to a very short list of the exact properties of this fundamental TDDFT/TDCDFT functional. In addition, we expect that our antiadiabatic kernel may become a useful tool for practical calculations of the absorption spectra in various many-body systems, in spite of the fact that its formal limit of applicability $(\omega \rightarrow \infty)$ is apparently outside the realistic range of excitation energies. As a matter of fact, the adiabatic xc kernel suffers from a very similar formal problem but still it is routinely used in most practical applications of TDDFT. It is also interesting to note a close physical similarity of the two limiting forms (adiabatic and antiadiabatic) of $f_{\mathrm{xc}}$. Physically both rely on a "collective" treatment of the $\mathrm{xc}$ effects. In the adiabatic case the collective motion is completely "relaxed" to the "instantaneous" ground state while in its antiadiabatic counterpart it is completely unrelaxed and can be viewed as a deformation of the initial ground state. Clearly the truth is in between, but at the current level of the theory there is no any clear argument in favor of one of those limits, except for the fact that the precise form of the antiadiabatic xc kernel has not been known so far. Our exact result for $f_{\mathrm{xc}}^{\infty}$ is a step toward the understanding of these matters.

This paper is organized as follows. In Sec. II we present a general formulation for the antiadiabatic tensor and scalar xc kernels in terms of the first spectral moment of the current density fluctuation spectrum. In Sec. III we provide explicit expressions for these kernels in finite or infinite systems in real space. In Sec. IV we make connection with the physically instructive continuum mechanics formulation and discuss the interpretation of $f_{\mathrm{xc}}$ in terms of elastic constant (stiffnesses) of the electronic system. In Sec. V we present the expressions for the tensor and scalar xc kernels for an infinite periodic system in momentum space. Working in momentum space allows us to focus most clearly on the long rangedness of the kernels in the $q \rightarrow 0$ limit: Sec. VI summarizes our results for the singular parts of the kernel. Finally, Appendix A presents a detailed derivation of the allimportant double commutator $M$, and Appendix B presents 
results for the longitudinal and transversal components of the antiadiabatic $\hat{f}_{\mathrm{xc}}$ in the homogeneous electron gas, obtained by using state-of-the-art values of the static structure factor and correlation energy.

\section{GENERAL FORMULATION}

The current response tensor can be written as

$$
\begin{aligned}
\chi_{\mu \nu}\left(\mathbf{r}, \mathbf{r}^{\prime}, \omega\right)= & \frac{e n_{0}(\mathbf{r}) \delta\left(\mathbf{r}-\mathbf{r}^{\prime}\right)}{c m} \delta_{\mu \nu} \\
& +\frac{e}{c} \sum_{\alpha, \beta}[F(\alpha)-F(\beta)] \frac{\left\langle\alpha\left|j_{\mu}(\mathbf{r})\right| \beta\right\rangle\left\langle\beta\left|j_{\nu}\left(\mathbf{r}^{\prime}\right)\right| \alpha\right\rangle}{\omega-E_{\beta}+E_{\alpha}+i \eta},
\end{aligned}
$$

where $|\alpha\rangle$ and $E_{\alpha}$ are the many-body wave functions and eigenenergies, respectively, $n_{0}(\mathbf{r})$ is the ground-state electron density, $\mathbf{j}(\mathbf{r})=\Sigma_{n}\left[\mathbf{p}_{n} \delta\left(\mathbf{r}-\mathbf{r}_{n}\right)+\delta\left(\mathbf{r}-\mathbf{r}_{n}\right) \mathbf{p}_{n}\right] /(2 m)$ is the paramagnetic current-density operator, $\mathbf{p}_{n}$ is the momentum operator of the $n$th electron, and $F(\alpha)$ are the diagonal elements of the equilibrium (or ground state at $T=0$ ) density matrix. Expansion of the right-hand side of Eq. (3) in $1 / \omega$ gives to the second order

$$
\chi_{\mu \nu}\left(\mathbf{r}, \mathbf{r}^{\prime}, \omega\right) \stackrel{\omega \rightarrow \infty}{\approx} \frac{e}{c}\left[\frac{n_{0}(\mathbf{r})}{m} \delta\left(\mathbf{r}-\mathbf{r}^{\prime}\right) \delta_{\mu \nu}+\frac{M_{\mu \nu}\left(\mathbf{r}, \mathbf{r}^{\prime}\right)}{m^{2} \omega^{2}}\right],
$$

where

$$
M_{\mu \nu}\left(\mathbf{r}, \mathbf{r}^{\prime}\right)=m^{2}\left\langle\left[j_{\mu}(\mathbf{r}),\left[H_{0}, j_{\nu}\left(\mathbf{r}^{\prime}\right)\right]\right]\right\rangle
$$

is the first moment of the imaginary part of $\chi_{\mu \nu}$, the angle brackets denoting the ground state or equilibrium average at zero or finite temperature, respectively,

$$
H_{0}=\sum_{n=1}^{N}\left[\frac{p_{n}^{2}}{2 m}+V_{0}\left(\mathbf{r}_{n}\right)\right]+\frac{1}{2} \sum_{n \neq m}^{N} V_{C}\left(\left|\mathbf{r}_{n}-\mathbf{r}_{m}\right|\right)
$$

is the Hamiltonian of the unperturbed system, $V_{0}(\mathbf{r})$ is the external potential and $N$ is the number of electrons in the system. Inversion of Eq. (4) yields

$$
\chi_{\mu \nu}^{-1}\left(\mathbf{r}, \mathbf{r}^{\prime}, \omega\right) \stackrel{\omega \rightarrow \infty}{\approx} \frac{c}{e}\left[\frac{m \delta\left(\mathbf{r}-\mathbf{r}^{\prime}\right)}{n_{0}(\mathbf{r})} \delta_{\mu \nu}-\frac{M_{\mu \nu}\left(\mathbf{r}, \mathbf{r}^{\prime}\right)}{\omega^{2} n_{0}(\mathbf{r}) n_{0}\left(\mathbf{r}^{\prime}\right)}\right] .
$$

Using the definition of the tensor exchange-correlation kernel of TDCDFT (Ref. 2) of Eq. (2) we can write

$$
f_{\mathrm{xc}, \mu \nu}\left(\mathbf{r}, \mathbf{r}^{\prime}, \omega\right) \stackrel{\omega \rightarrow \infty}{\approx} \frac{c M_{\mu \nu}^{\mathrm{xc}}\left(\mathbf{r}, \mathbf{r}^{\prime}\right)}{e \omega^{2} n_{0}(\mathbf{r}) n_{0}\left(\mathbf{r}^{\prime}\right)}
$$

where

$$
\begin{aligned}
M_{\mu \nu}^{\mathrm{xc}}\left(\mathbf{r}, \mathbf{r}^{\prime}\right)= & M_{\mu \nu}\left(\mathbf{r}, \mathbf{r}^{\prime}\right)-M_{\mu \nu}^{s}\left(\mathbf{r}, \mathbf{r}^{\prime}\right) \\
& -n_{0}(\mathbf{r}) n_{0}\left(\mathbf{r}^{\prime}\right) \partial_{\mu} \partial_{\nu}^{\prime} V_{C}\left(\left|\mathbf{r}-\mathbf{r}^{\prime}\right|\right) .
\end{aligned}
$$

From here forth, derivatives act on all the functions of corresponding variables to the right of them.

The connection to the scalar quantities follows from the equality

$$
\chi\left(\mathbf{r}, \mathbf{r}^{\prime}, \omega\right)=\frac{c \partial_{\mu} \partial_{\nu}^{\prime} \chi_{\mu \nu}\left(\mathbf{r}, \mathbf{r}^{\prime}, \omega\right)}{e \omega^{2}}
$$

where $\chi\left(\mathbf{r}, \mathbf{r}^{\prime}, \omega\right)$ is the density response function and the summation over the repeated coordinate indices is implied. By virtue of Eqs. (4) and (8) we can write

$$
\chi\left(\mathbf{r}, \mathbf{r}^{\prime}, \omega\right) \stackrel{\omega \rightarrow \infty}{\approx \omega^{2}} \partial_{\mu} n_{0}(\mathbf{r}) \partial_{\mu}^{\prime} \delta\left(\mathbf{r}-\mathbf{r}^{\prime}\right)+\frac{\partial_{\mu} \partial_{\nu}^{\prime} M_{\mu \nu}\left(\mathbf{r}, \mathbf{r}^{\prime}\right)}{m^{2} \omega^{4}}
$$

the inversion of which gives

$$
\begin{aligned}
\chi^{-1}\left(\mathbf{r}, \mathbf{r}^{\prime}, \omega\right) \approx & m \omega^{2} \mathcal{G}\left(\mathbf{r}, \mathbf{r}^{\prime}\right)-\int\left[\partial_{\mu}^{\prime \prime} \mathcal{G}\left(\mathbf{r}, \mathbf{r}^{\prime \prime}\right)\right] M_{\mu \nu}\left(\mathbf{r}^{\prime \prime}, \mathbf{r}^{\prime \prime \prime}\right) \\
& \times\left[\partial_{\nu}^{\prime \prime \prime} \mathcal{G}\left(\mathbf{r}^{\prime \prime \prime}, \mathbf{r}^{\prime}\right)\right] d \mathbf{r}^{\prime \prime} d \mathbf{r}^{\prime \prime \prime},
\end{aligned}
$$

where $\mathcal{G}\left(\mathbf{r}, \mathbf{r}^{\prime}\right)=\left[\partial_{\mu} n_{0}(\mathbf{r}) \partial_{\mu}^{\prime} \delta\left(\mathbf{r}-\mathbf{r}^{\prime}\right)\right]^{-1}$ can be written as the solution of the following equation:

$$
-\partial_{\mu} n_{0}(\mathbf{r}) \partial_{\mu} \mathcal{G}\left(\mathbf{r}, \mathbf{r}^{\prime}\right)=\delta\left(\mathbf{r}-\mathbf{r}^{\prime}\right) .
$$

With the use of the definition of the scalar xc kernel of Eq. (1) we have from Eqs. (10) and (7)

$$
f_{\mathrm{xc}}^{\infty}\left(\mathbf{r}, \mathbf{r}^{\prime}\right)=\int\left[\partial_{\mu}^{\prime \prime} \mathcal{G}\left(\mathbf{r}, \mathbf{r}^{\prime \prime}\right)\right] M_{\mu \nu}^{\mathrm{xc}}\left(\mathbf{r}^{\prime \prime}, \mathbf{r}^{\prime \prime \prime}\right)\left[\partial_{\nu}^{\prime \prime \prime} \mathcal{G}\left(\mathbf{r}^{\prime \prime \prime}, \mathbf{r}^{\prime}\right)\right] d \mathbf{r}^{\prime \prime} d \mathbf{r}^{\prime \prime \prime},
$$

where $f_{\mathrm{xc}}^{\infty}\left(\mathbf{r}, \mathbf{r}^{\prime}\right) \equiv \lim _{\omega \rightarrow \infty} f_{\mathrm{xc}}\left(\mathbf{r}, \mathbf{r}^{\prime}, \omega\right)$.

\section{TENSOR AND SCALAR XC KERNELS IN REAL SPACE}

In Appendix A, we outline a rather lengthy evaluation of the double commutator in Eq. (5) which leads to the following final expressions:

$$
\begin{aligned}
M_{\mu \nu}^{\mathrm{xc}}\left(\mathbf{r}, \mathbf{r}^{\prime}\right)= & T_{\alpha \alpha \mu \nu}^{\mathrm{xc}}\left(\mathbf{r}, \mathbf{r}^{\prime}\right)+T_{\nu \alpha \alpha \mu}^{\mathrm{xc}}\left(\mathbf{r}, \mathbf{r}^{\prime}\right)+T_{\alpha \mu \alpha \nu}^{\mathrm{xc}}\left(\mathbf{r}, \mathbf{r}^{\prime}\right) \\
& +\delta\left(\mathbf{r}-\mathbf{r}^{\prime}\right)\left[\int K_{\mu \nu}^{\mathrm{xc}}\left(\mathbf{r}, \mathbf{r}^{\prime \prime}\right) d \mathbf{r}^{\prime \prime}-n_{0}(\mathbf{r}) \partial_{\mu} \partial_{\nu} V_{\mathrm{xc}}(\mathbf{r})\right] \\
& -K_{\mu \nu}^{\mathrm{xc}}\left(\mathbf{r}, \mathbf{r}^{\prime}\right)
\end{aligned}
$$

where

$$
\begin{gathered}
T_{\alpha \beta \mu \nu}^{\mathrm{xc}}\left(\mathbf{r}, \mathbf{r}^{\prime}\right)=\partial_{\alpha} \partial_{\beta}^{\prime} \delta\left(\mathbf{r}-\mathbf{r}^{\prime}\right) T_{\mu \nu}^{\mathrm{xc}}(\mathbf{r}), \\
T_{\mu \nu}^{\mathrm{xc}}(\mathbf{r})=\left.\frac{1}{2 m}\left(\partial_{\mu} \partial_{\nu}^{\prime}+\partial_{\mu}^{\prime} \partial_{\nu}\right)\left[\rho_{1}\left(\mathbf{r}, \mathbf{r}^{\prime}\right)-\rho_{1 s}\left(\mathbf{r}, \mathbf{r}^{\prime}\right)\right]\right|_{\mathbf{r}^{\prime}=\mathbf{r}},
\end{gathered}
$$

$\rho_{1}\left(\mathbf{r}, \mathbf{r}^{\prime}\right)$ is ground-state (equilibrium) one-particle density matrix,

$$
K_{\mu \nu}^{\mathrm{xc}}\left(\mathbf{r}, \mathbf{r}^{\prime}\right)=n_{0}(\mathbf{r}) n_{0}\left(\mathbf{r}^{\prime}\right)\left[g\left(\mathbf{r}, \mathbf{r}^{\prime}\right)-1\right] \partial_{\mu} \partial_{\nu} V_{C}\left(\left|\mathbf{r}-\mathbf{r}^{\prime}\right|\right),
$$

$g\left(\mathbf{r}, \mathbf{r}^{\prime}\right)$ is the ground-state (equilibrium) pair-correlation function defined as $^{22}$ 


$$
g\left(\mathbf{r}, \mathbf{r}^{\prime}\right)=\frac{N(N-1)}{n_{0}(\mathbf{r}) n_{0}\left(\mathbf{r}^{\prime}\right)}\left\langle\delta\left(\mathbf{r}-\mathbf{r}_{1}\right) \delta\left(\mathbf{r}^{\prime}-\mathbf{r}_{2}\right)\right\rangle,
$$

where $\mathbf{r}_{1}$ and $\mathbf{r}_{2}$ are the coordinates of two distinct electrons of the system and $V_{\mathrm{xc}}(\mathbf{r})$ is the static xc potential.

For the scalar $f_{\mathrm{xc}}$, the substitution of Eq. (13) into Eq. (12) leads to

$$
\begin{aligned}
f_{\mathrm{xc}}^{\infty}\left(\mathbf{r}, \mathbf{r}^{\prime}\right)= & \int 3\left[\partial_{\alpha}^{\prime \prime} \partial_{\mu}^{\prime \prime} \mathcal{G}\left(\mathbf{r}, \mathbf{r}^{\prime \prime}\right)\right] T_{\mu \nu}^{\mathrm{xc}}\left(\mathbf{r}^{\prime \prime}\right)\left[\partial_{\alpha}^{\prime \prime} \partial_{\nu}^{\prime \prime} \mathcal{G}\left(\mathbf{r}^{\prime \prime}, \mathbf{r}^{\prime}\right)\right] d \mathbf{r}^{\prime \prime} \\
& +\int\left[\partial_{\mu}^{\prime \prime} \mathcal{G}\left(\mathbf{r}, \mathbf{r}^{\prime \prime}\right)\right] \Omega_{\mu \nu}^{\mathrm{xc}}\left(\mathbf{r}^{\prime \prime}\right)\left[\partial_{\nu}^{\prime \prime} \mathcal{G}\left(\mathbf{r}^{\prime \prime}, \mathbf{r}^{\prime}\right)\right] d \mathbf{r}^{\prime \prime} \\
& -\int\left[\partial_{\mu}^{\prime \prime} \mathcal{G}\left(\mathbf{r}, \mathbf{r}^{\prime \prime}\right)\right] K_{\mu \nu}^{\mathrm{xc}}\left(\mathbf{r}^{\prime \prime}, \mathbf{r}^{\prime \prime \prime}\right)\left[\partial_{\nu}^{\prime \prime \prime} \mathcal{G}\left(\mathbf{r}^{\prime \prime \prime}, \mathbf{r}^{\prime}\right)\right] d \mathbf{r}^{\prime \prime} d \mathbf{r}^{\prime \prime \prime},
\end{aligned}
$$

where an effective "xc curvature" $\Omega_{\mu \nu}^{\mathrm{xc}}(\mathbf{r})$ is defined as

$$
\Omega_{\mu \nu}^{\mathrm{xc}}(\mathbf{r})=\int K_{\mu \nu}^{\mathrm{xc}}\left(\mathbf{r}, \mathbf{r}^{\prime}\right) d \mathbf{r}^{\prime}-n_{0}(\mathbf{r}) \partial_{\mu} \partial_{\nu} V_{\mathrm{xc}}(\mathbf{r}) .
$$

\section{FORMULATION IN TERMS OF CONTINUUM MECHANICS}

In spite of their complicated appearance, the physical significance of formulas (13)-(19) is quite transparent. In fact, the formulas could be derived more quickly starting from the representation of $M_{\mu \nu}^{\mathrm{xc}}\left(\mathbf{r}, \mathbf{r}^{\prime}\right)$ as the second functional derivative of the exchange-correlation energy functional $E_{\mathrm{xc}}[\mathbf{u}]$ with respect to virtual displacements, $u_{\mu}(\mathbf{r})$ and $u_{\nu}\left(\mathbf{r}^{\prime}\right)$. The explicit expression for $E_{\mathrm{xc}}[\mathbf{u}]$ can be easily obtained from the formulas given in Refs. 18 and 19 for the full energy functional $E[\mathbf{u}]$ (kinetic plus Coulomb interaction plus external potential energy) by subtracting the noninteracting kinetic energy functional, the Hartree energy functional, and the Kohn-Sham potential energy. The main idea behind this "continuum mechanics" approach is to introduce a deformed ground-state wave function ${ }^{23}$

$$
\begin{aligned}
& \psi_{0}[\mathbf{u}]\left(\mathbf{r}_{1}, \ldots, \mathbf{r}_{N}\right) \\
& \quad \equiv \psi_{0}\left[\mathbf{r}_{1}-\mathbf{u}\left(\mathbf{r}_{1}\right), \ldots, \mathbf{r}_{N}-\mathbf{u}\left(\mathbf{r}_{N}\right)\right] \prod_{i=1}^{N} g^{-1 / 4}\left(\mathbf{r}_{i}\right),
\end{aligned}
$$

where the factors $g^{-1 / 4}$ ensure the correct normalization of the deformed state, $g^{1 / 2}$ being the Jacobian of the non volume-preserving transformation of coordinates $\mathbf{r} \rightarrow \mathbf{r}$ $-\mathbf{u}(\mathbf{r})$, i.e., the determinant of the matrix $\delta_{\mu \nu}-\partial_{\mu} u_{v}$. Then we have

$$
M_{\mu \nu}\left(\mathbf{r}, \mathbf{r}^{\prime}\right)=\left.\frac{\delta^{2} E[u]}{\delta u_{\mu}(\mathbf{r}) \delta u_{\nu}\left(\mathbf{r}^{\prime}\right)}\right|_{\mathbf{u}=0},
$$

where $E[\mathbf{u}]$ is the expectation value of the Hamiltonian in the deformed ground state $\psi_{0}[\mathbf{u}]$. Similarly, we have

$$
M_{\mu \nu}^{\mathrm{xc}}\left(\mathbf{r}, \mathbf{r}^{\prime}\right)=\left.\frac{\delta^{2} E_{\mathrm{xc}}[\mathbf{u}]}{\delta u_{\mu}(\mathbf{r}) \delta u_{\nu}\left(\mathbf{r}^{\prime}\right)}\right|_{\mathbf{u}=0},
$$

where, as noted above, $E_{\mathrm{xc}}[\mathbf{u}]$ is defined as the difference $E[\mathbf{u}]-E_{H}[\mathbf{u}]-T_{s}[\mathbf{u}]-V_{s}[\mathbf{u}]$, where $E_{H}[\mathbf{u}]$ is the Hartree energy, $T_{s}[\mathbf{u}]$ is the kinetic energy of the deformed Kohn-Sham ground state (i.e., the noninteracting ground state that has the same density as the true ground state) and $V_{s}[\mathbf{u}]$ is the KohnSham potential energy in the deformed ground state.

The above expressions are very helpful in understanding the physical significance of the terms that appear in the evaluation of the double commutator in Eq. (5). To second order in $\mathbf{u}$ the $\mathrm{xc}$ energy functional has the form ${ }^{18,19}$

$$
\begin{aligned}
E_{\mathrm{xc}}[\mathbf{u}]= & \frac{1}{2} \int d \mathbf{r}\left\{-u_{\mu}(\mathbf{r}) u_{\nu}(\mathbf{r}) n_{0}(\mathbf{r}) \partial_{\mu} \partial_{\nu} V_{\mathrm{xc}}(\mathbf{r})+T_{\mu \nu}^{\mathrm{xc}}(\mathbf{r})\right. \\
& \left.\times\left\{4 u_{\mu \alpha}(\mathbf{r}) u_{\nu \alpha}(\mathbf{r})-\left[\partial_{\mu} u_{\alpha}(\mathbf{r})\right]\left[\partial_{\nu} u_{\alpha}(\mathbf{r})\right]\right\}\right\} \\
& +\frac{1}{4} \int d \mathbf{r} d \mathbf{r}^{\prime}\left[u_{\mu}(\mathbf{r})-u_{\mu}\left(\mathbf{r}^{\prime}\right)\right] \\
& \times K_{\mu \nu}^{\mathrm{xc}}\left(\mathbf{r}, \mathbf{r}^{\prime}\right)\left[u_{\nu}(\mathbf{r})-u_{\nu}\left(\mathbf{r}^{\prime}\right)\right],
\end{aligned}
$$

where $T_{\mu \nu}^{\mathrm{xc}}(\mathbf{r})$ and $K_{\mu \nu}^{\mathrm{xc}}\left(\mathbf{r}, \mathbf{r}^{\prime}\right)$ are defined by Eqs. (15) and (16), respectively. The second term in Eq. (23) is a typical energy of an elastic medium, which is quadratic in the deformation gradients (strains). ${ }^{24}$ The corresponding elastic moduli are determined by the xc kinetic stress tensor $T_{\mu \nu}^{\mathrm{xc}}$ The third term is an additional nonlocal contribution which should be present if the continuum is charged and the displacement produces a local polarization. Hence the third term corresponds to the xc part of the dipole-dipole interaction energy of an inhomogeneously polarized continuum. Apparently the first two terms vanish in the case of a homogeneous (rigid) displacement since it does not cause deformations and thus does not cost any energy. For the rigid displacement only the first term in Eq. (23) survives. This term ensures the harmonic potential theorem. It cancels the $\mathrm{xc}$ part of the KS potential, which guaranties that the rigid motion of the whole system is controlled solely by the external potential.

The above expression for $E_{\mathrm{xc}}$ can be rewritten in terms of the effective curvature $\Omega_{\mu \nu}^{\mathrm{xc}}(\mathbf{r})$, defined in Eq. (19), and we get

$$
\begin{aligned}
E_{\mathrm{xc}}[\mathbf{u}]= & -\frac{1}{2} \int d \mathbf{r} d \mathbf{r}^{\prime} u_{\mu}(\mathbf{r}) K_{\mu \nu}^{\mathrm{xc}}\left(\mathbf{r}, \mathbf{r}^{\prime}\right) u_{\nu}\left(\mathbf{r}^{\prime}\right) \\
& +\frac{1}{2} \int d \mathbf{r}\left\{\Omega_{\mu \nu}^{\mathrm{xc}} u_{\mu} u_{\nu}+T_{\mu \nu}^{\mathrm{xc}}\left[4 u_{\mu \alpha} u_{\nu \alpha}-\left(\partial_{\mu} u_{\alpha}\right)\left(\partial_{\nu} u_{\alpha}\right)\right]\right\}
\end{aligned}
$$

from which the expression (13) for $M_{\text {xc }}$ can be straightforwardly recovered by isolating the coefficient of $u_{\mu}(\mathbf{r}) u_{\nu}\left(\mathbf{r}^{\prime}\right)$ (this requires some integrations by parts).

\section{APPLICATION TO THE PERIODIC CASE}

In the case of a periodic system, the response functions and xc kernels become infinite matrices indexed by recipro- 
cal lattice vectors. The Fourier transform of Eq. (6) gives

$f_{\mathbf{G G}^{\prime}, \mu \nu}^{\mathrm{xc}}(\mathbf{q}, \omega) \stackrel{\omega \rightarrow \infty}{\approx} \frac{c}{e \omega^{2}} n_{0}^{-1}\left(\mathbf{G}-\mathbf{G}^{\prime \prime}\right) M_{\mathbf{G}^{\prime \prime} \mathbf{G}^{\prime \prime \prime}, \mu \nu}^{\mathrm{xc}}(\mathbf{q}) n_{0}^{-1}\left(\mathbf{G}^{\prime \prime \prime}-\mathbf{G}^{\prime}\right)$,

where $n_{0}^{-1}(\mathbf{G})$ is the Fourier transform of $1 / n_{0}(\mathbf{r})$ and we imply summation over the repeated reciprocal vectors. By Eq. (13), we can write in the reciprocal space

$$
\begin{aligned}
M_{\mathbf{G G}^{\prime}, \mu \nu}^{\mathrm{xc}}(\mathbf{q})= & (\mathbf{G}+\mathbf{q}) \cdot\left(\mathbf{G}^{\prime}+\mathbf{q}\right) T_{\mu \nu}^{\mathrm{xc}}\left(\mathbf{G}-\mathbf{G}^{\prime}\right) \\
& +\left(G_{\alpha}^{\prime}+q_{\alpha}\right) T_{\alpha \mu}^{\mathrm{xc}}\left(\mathbf{G}-\mathbf{G}^{\prime}\right)\left(G_{\nu}+q_{\nu}\right)+\left(G_{\mu}^{\prime}+q_{\mu}\right) \\
& \times\left(G_{\alpha}+q_{\alpha}\right) T_{\alpha \nu}^{\mathrm{xc}}\left(\mathbf{G}-\mathbf{G}^{\prime}\right)-\left[K_{\mathbf{G G}^{\prime}, \mu \nu}^{\mathrm{xc}}(\mathbf{q})\right. \\
& \left.-K_{\mathbf{G} 0, \mu \nu}^{\mathrm{xc}}(0) \delta_{0 \mathbf{G}^{\prime}}\right]+\left(G_{\mu}^{\prime}-G_{\mu}^{\prime \prime}\right)\left(G_{\nu}^{\prime}-G_{\nu}^{\prime \prime}\right) \\
& \times n_{0}\left(\mathbf{G}-\mathbf{G}^{\prime \prime}\right) V_{\mathrm{xc}}\left(\mathbf{G}^{\prime \prime}-\mathbf{G}^{\prime}\right),
\end{aligned}
$$

where $T_{\mu \nu}^{\mathrm{xc}}(\mathbf{G})$ is the Fourier transform of $T_{\mu \nu}^{\mathrm{xc}}(\mathbf{r})$ and $K_{\mathbf{G G}^{\prime}, \mu \nu}^{\mathrm{xc}}(\mathbf{q})$ is the double Fourier transform of $K_{\mu \nu}^{\mathrm{xc}}\left(\mathbf{r}, \mathbf{r}^{\prime}\right)$.

We can write

$$
f_{\mathbf{G G}^{\prime}}^{\mathrm{xc}, \infty}(\mathbf{q})=\mathcal{G}_{\mathbf{G G}^{\prime \prime}}(\mathbf{q}) M_{\mathbf{G}^{\prime \prime} \mathbf{G}^{\prime \prime \prime}}^{\mathrm{xc}}(\mathbf{q}) \mathcal{G}_{\mathbf{G}^{\prime \prime \prime} \mathbf{G}^{\prime}}(\mathbf{q})
$$

where

$$
\begin{aligned}
M_{\mathbf{G G}^{\prime}}^{\mathrm{xc}}(\mathbf{q})= & \left(G_{\mu}+q_{\mu}\right) M_{\mathbf{G G}^{\prime}, \mu \nu}^{\mathrm{xc}}(\mathbf{q})\left(G_{\nu}^{\prime}+q_{\nu}\right)=\left(G_{\mu}+q_{\mu}\right) \\
& \times\left\{3(\mathbf{G}+\mathbf{q}) \cdot\left(\mathbf{G}^{\prime}+\mathbf{q}\right) T_{\mu \nu}^{\mathrm{xc}}\left(\mathbf{G}-\mathbf{G}^{\prime}\right)\right. \\
& -\left[K_{\mathbf{G G}^{\prime}, \mu \nu}^{\mathrm{xc}}(\mathbf{q})-K_{\mathbf{G} 0, \mu \nu}^{\mathrm{xc}}(0) \delta_{0 \mathbf{G}^{\prime}}\right]+\left(G_{\mu}^{\prime}-G_{\mu}^{\prime \prime}\right) \\
& \left.\times\left(G_{\nu}^{\prime}-G_{\nu}^{\prime \prime}\right) n_{0}\left(\mathbf{G}-\mathbf{G}^{\prime \prime}\right) V_{\mathrm{xc}}\left(\mathbf{G}^{\prime \prime}-\mathbf{G}^{\prime}\right)\right\}\left(G_{\nu}^{\prime}+q_{\nu}\right) .
\end{aligned}
$$

\section{LONG-WAVELENGTH LIMIT}

Because of the applications in optics and because of the singularities arising in the scalar $f^{\mathrm{xc}}, 9$ the long-wave limit $(\mathbf{q} \rightarrow 0)$ requires a separate consideration. By the structure of Eq. (26), $M_{\mathbf{G G}^{\prime}, \mu \nu}^{\mathrm{xc}}(\mathbf{q})$ have finite limit as $\mathbf{q} \rightarrow 0$. By Eq. (25), the same is true for $f_{\mathbf{G G}^{\prime}, \mu \nu}^{\mathrm{xc}}(\mathbf{q})$. We note that in the long-wave limit the "head" and "wing" elements of $M_{\mathbf{G G}^{\prime}, \mu \nu}^{\mathrm{xc}}$ reduce to

$$
M_{\mathbf{G} 0, \mu \nu}^{\mathrm{xc}}=M_{0,-\mathbf{G}, \nu \mu}^{\mathrm{xc}}=G_{\mu}^{\prime \prime} G_{\nu}^{\prime \prime} n_{0}\left(\mathbf{G}-\mathbf{G}^{\prime \prime}\right) V_{\mathrm{xc}}\left(\mathbf{G}^{\prime \prime}\right),
$$

i.e., they are expressed in terms of the ground-state density and xc potential only. Equation (25), however, shows that this is not the case with $f_{00, \mu \nu}^{\mathrm{xc}}$ and $f_{\mathbf{G} \neq 0,0, \mu \nu}^{\mathrm{xc}}$, for the knowledge of which $T_{\mathbf{G G}^{\prime}, \mu \nu}^{\mathrm{xc}}$ and $K_{\mathbf{G G}^{\prime}, \mu \nu}^{\mathrm{xc}}$ are needed.

For the scalar $f^{\mathrm{xc}}$, it can be, however, noticed that Eq. (27) gives a singular long-wave limit. Indeed, since $\mathcal{G}_{\mathbf{G G}^{\prime}}(\mathbf{q})$ $=\mathcal{N}_{\mathbf{G G}^{\prime}}^{-1}(\mathbf{q})$, where

$$
\mathcal{N}_{\mathbf{G G}^{\prime}}(\mathbf{q})=(\mathbf{G}+\mathbf{q}) \cdot\left(\mathbf{G}^{\prime}+\mathbf{q}\right) n_{0}\left(\mathbf{G}-\mathbf{G}^{\prime}\right)
$$

and the upper row (left column) of $\mathcal{N}_{\mathbf{G G}^{\prime}}(\mathbf{q})$ is zero in the $\mathbf{q} \rightarrow 0$ limit, $\mathcal{G}_{\mathbf{G G}^{\prime}}(\mathbf{q})$ is singular in this limit. To isolate the singularity, we introduce a matrix

$$
\tilde{\mathcal{N}}_{\mathbf{G G}^{\prime}}(\mathbf{q})=\frac{\mathcal{N}_{\mathbf{G G}^{\prime}}(\mathbf{q})}{|\mathbf{G}+\mathbf{q}|\left|\mathbf{G}^{\prime}+\mathbf{q}\right|}
$$

the inverse of which is regular at $\mathbf{q} \rightarrow 0$. We can write

$$
f_{\mathbf{G G}^{\prime}}^{\mathrm{xc}, \infty}(\mathbf{q})=\frac{\tilde{\mathcal{N}}_{\mathbf{G G}^{\prime \prime}}^{-1}(\mathbf{q}) M_{\mathbf{G}^{\prime \prime} \mathbf{G}^{\prime \prime \prime}}^{\mathrm{xc}}(\mathbf{q}) \tilde{\mathcal{N}}_{\mathbf{G}^{\prime \prime \prime} \mathbf{G}^{\prime}}^{-1}(\mathbf{q})}{\left|\mathbf{G}+\mathbf{q}\left\|\mathbf{G}^{\prime \prime}+\mathbf{q}\right\| \mathbf{G}^{\prime \prime \prime}+\mathbf{q} \| \mathbf{G}^{\prime}+\mathbf{q}\right|} .
$$

Retaining the leading terms in $1 / q$ only, we have in the long-wave limit

$$
f_{00}^{\mathrm{xc}, \infty}(\mathbf{q} \rightarrow 0)=\frac{\alpha}{q^{2}},
$$

where

$$
\begin{aligned}
& \alpha=\left[\tilde{\mathcal{N}}_{00}^{-1}(\hat{\mathbf{q}})\right]^{2} \hat{q}_{\mu} M_{00, \mu \nu}^{\mathrm{xc}} \hat{q}_{\nu}+2 \tilde{\mathcal{N}}_{00}^{-1}(\hat{\mathbf{q}}) \sum_{\mathbf{G} \neq 0} \tilde{\mathcal{N}}_{0 \mathbf{G}}^{-1}(\hat{\mathbf{q}}) \hat{G}_{\mu} M_{\mathbf{G} 0, \mu \nu}^{\mathrm{xc}} \hat{q}_{\nu} \\
& +\sum_{\mathbf{G}, \mathbf{G}^{\prime} \neq 0} \tilde{\mathcal{N}}_{0 \mathbf{G}}^{-1}(\hat{\mathbf{q}}) \hat{G}_{\mu} M_{\mathbf{G G}^{\prime}, \mu \nu}^{\mathrm{xc}} \hat{G}_{\nu}^{\prime} \tilde{\mathcal{N}}_{\mathbf{G}^{\prime} 0}^{-1}(\hat{\mathbf{q}}), \\
& f_{\mathbf{G} \neq 0,0}^{\mathrm{xc}, \infty}(\mathbf{q} \rightarrow 0)=f_{0,-\mathbf{G}}^{\mathrm{xc}, \infty}(\mathbf{q} \rightarrow 0) \\
& =\frac{1}{q G}\left\{\tilde{\mathcal{N}}_{\mathbf{G} 0}^{-1}(\hat{\mathbf{q}}) \hat{q}_{\mu} M_{00, \mu \nu}^{\mathrm{xc}} \hat{q}_{\nu} \tilde{\mathcal{N}}_{00}^{-1}(\hat{\mathbf{q}})\right. \\
& +\sum_{\mathbf{G}^{\prime} \neq 0}\left[\tilde{\mathcal{N}}_{\mathbf{G G}^{\prime}}^{-1}(\hat{\mathbf{q}}) \tilde{\mathcal{N}}_{00}^{-1}(\hat{\mathbf{q}})\right. \\
& \left.+\tilde{\mathcal{N}}_{\mathbf{G} 0}^{-1}(\hat{\mathbf{q}}) \tilde{\mathcal{N}}_{\mathbf{G}^{\prime} 0}^{-1}(\hat{\mathbf{q}})\right] \hat{G}_{\mu}^{\prime} M_{\mathbf{G}^{\prime} 0, \mu \nu}^{\mathrm{xc}} \hat{q}_{\nu} \\
& \left.+\sum_{\mathbf{G}^{\prime}, \mathbf{G}^{\prime \prime} \neq 0} \tilde{\mathcal{N}}_{\mathbf{G G}^{\prime}}^{-1}(\hat{\mathbf{q}}) \hat{G}_{\mu}^{\prime} M_{\mathbf{G}^{\prime} \mathbf{G}^{\prime \prime}, \mu \nu}^{\mathrm{xc}} \hat{G}_{\nu}^{\prime \prime} \tilde{\mathcal{N}}_{\mathbf{G}^{\prime \prime} 0}^{-1}(\hat{\mathbf{q}})\right\}, \\
& f_{\mathbf{G} \neq 0, \mathbf{G}^{\prime} \neq 0}^{\mathrm{xc}, \infty}(\mathbf{q} \rightarrow 0)=\frac{1}{G G^{\prime}}\left\{\tilde{\mathcal{N}}_{\mathbf{G} 0}^{-1}(\hat{\mathbf{q}}) \hat{q}_{\mu} M_{00, \mu \nu}^{\mathrm{xc}} \hat{q}_{\nu} \tilde{\mathcal{N}}_{0 \mathbf{G}^{\prime}}^{-1}(\hat{\mathbf{q}})\right. \\
& +\sum_{\mathbf{G}^{\prime \prime} \neq 0}\left[\tilde{\mathcal{N}}_{\mathbf{G G}^{\prime \prime}}^{-1}(\hat{\mathbf{q}}) \tilde{\mathcal{N}}_{0 \mathbf{G}^{\prime}}^{-1}(\hat{\mathbf{q}})\right. \\
& \left.+\tilde{\mathcal{N}}_{\mathbf{G} 0}^{-1}(\hat{\mathbf{q}}) \tilde{\mathcal{N}}_{\mathbf{G}^{\prime \prime} \mathbf{G}^{\prime}}^{-1}(\hat{\mathbf{q}})\right] \hat{G}_{\mu}^{\prime \prime} M_{\mathbf{G}^{\prime \prime} 0, \mu \nu}^{\mathrm{xc}} \hat{q}_{\nu} \\
& \left.+\sum_{\mathbf{G}^{\prime \prime}, \mathbf{G}^{\prime \prime \prime} \neq 0} \tilde{\mathcal{N}}_{\mathbf{G}^{\prime} \mathbf{G}^{\prime \prime}}^{-1}(\hat{\mathbf{q}}) \hat{G}_{\mu}^{\prime \prime} M_{\mathbf{G}^{\prime \prime} \mathbf{G}^{\prime \prime \prime}, \mu \nu}^{\mathrm{xc}} \hat{G}_{\nu}^{\prime \prime \prime} \tilde{\mathcal{N}}_{\mathbf{G}^{\prime \prime \prime} \mathbf{G}^{\prime}}^{-1}(\hat{\mathbf{q}})\right\}
\end{aligned}
$$

$M_{\mathbf{G G}^{\prime}, \mu \nu}^{\mathrm{xc}}$ are taken at $\mathbf{q}=0$ and vectors with a hat denote unit vectors in the same direction.

The singularity in $q$ of the head and wings elements of the scalar $f^{\mathrm{xc}}$ in the long-wave limit has an important physical implication: it is directly relevant to the description of the excitonic effect in semiconductors and insulators by means of TDDFT. ${ }^{9,10,25}$ We point out that this singularity is entirely due to the inhomogeneity since it disappears in the homogeneous case when the nondiagonal elements of the matrices are zero (cf. Ref. 17).

\section{SUMMARY AND CONCLUSIONS}

In the high-frequency limit, we have derived the exact representation of the xc kernel of an inhomogeneous manybody system in terms of the ground-state (or equilibrium at finite temperature) properties of the system. Both the scalar xc kernel $f_{\mathrm{xc}}$ and tensor xc kernel $\hat{f}_{\mathrm{xc}}$, relevant to the time- 
dependent density-functional theory and the time-dependent current-density functional theory, respectively, have been worked out.

The ground-state properties which define the infinitefrequency xc kernel have been found to be: (i) particle density; (ii) the kinetic exchange-correlation stress tensor; (iii) the pair-correlation function; and (iv) the exchangecorrelation potential. The former three can be found, in principle, exactly, by the method of the quantum Monte Carlo, while the latter can be obtained by the inversion of the KohnSham equations after the ground-state density has been found.

Keeping in view applications to finite and nonperiodic infinite systems, we have worked out the real-space form of the high-frequency xc kernels. The real-space formulation is most directly connected with the quantum continuum mechanics formulation of Refs. 18 and 19, and clearly displays the physical meaning of the antiadiabatic $f_{\mathrm{xc}}$ as elastic constants (stiffnesses) of the system.

In the periodic case and in the long-wave limit, our results contain an important singularity in the scalar $f_{\mathrm{xc}}$ as a function of the wave vector. This singularity is known to be a manifestation of the ultra nonlocality of $f_{\mathrm{xc}}$ in space and its presence is crucial for reproducing the excitonic effect in semiconductors and insulators by means of TDDFT.

We expect the results of this work to facilitate the construction of exchange-correlation kernels of TDDFT and TDCDFT, accurate enough to account for the many-body effects in the linear-response theory with applications to optics, electron energy-loss spectroscopy, and other fields. First of all one may expect that a direct application of our antiadiabatic kernel to the calculation of the excitation energies will give already reasonable results. Having now at hand a fully nonlocal xc kernel it is extremely interesting to test its performance and to compare the results with those obtained by using the common adiabatic $f_{\mathrm{xc}}$. Currently the antiadiabatic and the adiabatic limiting forms of the true dynamical xc kernel look almost equidistant from reality. An encouraging observation is that they are formally very similar, being instantaneous functionals of the displacement and the density, respectively. Both adiabatic and antiadiabatic kernels also involve similar physical assumptions, amounting to a collective treatment of the xc effects. The difference is that in the former case the collective motion is fully relaxed to the instantaneous ground state while it is completely unrelaxed in the latter limit. Which type of motion better fits to the real world is an open question that is now close to its final resolution. Of course, a possible answer to this question is that neither is sufficiently good. However, even in this case our exact results open a way for constructing more elaborate dynamical approximations by interpolating between the limiting forms of the kernel, for which $f_{\mathrm{xc}}^{\infty}$ is a necessary ingredient. Unfortunately the knowledge of only the two limits is not sufficient for a straightforward and accurate interpolation and in practice it will require considerable work. Hence we did not touch this problem in the present paper.

In this work we were mainly concerned with the derivation of the exact results and therefore we were working with the formally exact description of both the interacting and the $\mathrm{KS}$ systems. From the point of view of the practical imple- mentation this means that all our formulas are strictly valid for the all-electrons implementation of DFT. Frequently one wants to reduce the amount of computational work by treating explicitly only valence electrons and using pseudopotentials. In this case one should be careful because the presence of nonlocal pseudopotentials modifies the form of the current operator and therefore some explicit formulas may acquire a corresponding correction. Obviously the general results of Eqs. (6) and (12) are universal while the explicit form of the xc stress tensors entering Eq. (18) will be modified depending on a form of pseudopotentials used in a particular implementation. These corrections can be found straightforwardly along the same lines as in the exact formulation of the theory in this paper.

\section{ACKNOWLEDGMENTS}

This work was supported by DOE under Grant No. DEFG02-05ER46203 (G.V., S.P.). G.V. gratefully acknowledges the hospitality of the ETSF in San Sebastian where this work was initiated and the Institute for Solid State Physics of the University of Tokyo where it was completed. V.U.N. gratefully acknowledges the hospitality of the University of Missouri-Columbia. I.V.T. acknowledges support by the Spanish MEC (Grant No. FIS2007-65702-C02-01), ACIPromociona (Grant No. ACI2009-1036), Grupos Consolidados UPV/EHU del Gobierno Vasco (Grant No. IT-319-07), and the European Union through e-I3 ETSF project (Contract No. 211956).

\section{APPENDIX A: DERIVATION OF $M_{\mu \nu}^{\mathrm{xc}}\left(\mathbf{r}, \mathbf{r}^{\prime}\right)$}

In this appendix, we outline the derivation of the expectation values of the commutators relevant to the evaluation of the moment of Eq. (5). Since the system is assumed paramagnetic, the ground-state (equilibrium) expectation value of the current is set to zero throughout.

(i) Kinetic energy part. A straightforward evaluation leads to

$$
\begin{aligned}
\frac{m}{2}\left\langle\left[j_{n \mu}(\mathbf{r}),\left[p_{n}^{2}, j_{n \nu}\left(\mathbf{r}^{\prime}\right)\right]\right]\right\rangle \\
=\partial_{\alpha}^{\prime} \partial_{\alpha} \delta\left(\mathbf{r}-\mathbf{r}^{\prime}\right)\left\langle p_{n \mu} j_{n \nu}(\mathbf{r})\right\rangle+\partial_{\alpha}^{\prime} \partial_{\nu} \delta\left(\mathbf{r}-\mathbf{r}^{\prime}\right)\left\langle p_{n \alpha} j_{n \mu}(\mathbf{r})\right\rangle \\
\quad-\partial_{\alpha}^{\prime} \partial_{\mu}^{\prime} \delta\left(\mathbf{r}-\mathbf{r}^{\prime}\right)\left\langle p_{n \alpha} j_{n \nu}(\mathbf{r})\right\rangle \\
\quad+\frac{1}{4 m} \partial_{\alpha}^{\prime} \partial_{\nu} \partial_{\mu}^{\prime} \partial_{\alpha} \delta\left(\mathbf{r}-\mathbf{r}^{\prime}\right)\left\langle\delta\left(\mathbf{r}-\mathbf{r}_{n}\right)\right\rangle .
\end{aligned}
$$

Introducing a tensor

$$
T_{\mu \nu}(\mathbf{r})=\frac{1}{2} \sum_{n}\left\langle p_{n \mu} j_{n \nu}(\mathbf{r})+p_{n \nu} j_{n \mu}(\mathbf{r})\right\rangle
$$

we express it as

$$
T_{\mu \nu}(\mathbf{r})=-\frac{1}{m}-\left.\partial_{\mu}^{\prime} \partial_{\nu}^{\prime} \rho_{1}\left(\mathbf{r}, \mathbf{r}^{\prime}\right)\right|_{\mathbf{r}^{\prime}=\mathbf{r}}+\frac{1}{2 m} \partial_{\mu} \partial_{\nu} n_{0}(\mathbf{r}),
$$

where $\rho_{1}\left(\mathbf{r}, \mathbf{r}^{\prime}\right)$ is the ground-state (equilibrium) one particle density matrix. For the kinetic energy part we, therefore, have 


$$
\begin{aligned}
m^{2}\langle & {\left.\left[j_{\mu}(\mathbf{r}),\left[T, j_{\nu}\left(\mathbf{r}^{\prime}\right)\right]\right]\right\rangle } \\
= & \partial_{\alpha} \partial_{\alpha}^{\prime} \delta\left(\mathbf{r}-\mathbf{r}^{\prime}\right) T_{\mu \nu}(\mathbf{r})+\partial_{\alpha}^{\prime} \partial_{\nu} \delta\left(\mathbf{r}-\mathbf{r}^{\prime}\right) T_{\alpha \mu}(\mathbf{r}) \\
& \quad-\partial_{\mu}^{\prime} \partial_{\alpha}^{\prime} \delta\left(\mathbf{r}-\mathbf{r}^{\prime}\right) T_{\alpha \nu}(\mathbf{r}) \\
& +\frac{1}{4 m} \partial_{\alpha}^{\prime} \partial_{\nu} \partial_{\mu}^{\prime} \partial_{\alpha} \delta\left(\mathbf{r}-\mathbf{r}^{\prime}\right) n_{0}(\mathbf{r})
\end{aligned}
$$

(ii) The external potential part is easily evaluated to

$m^{2}\left\langle\left[j_{\mu}(\mathbf{r}),\left[\sum_{n} V_{0}\left(\mathbf{r}_{n}\right), j_{\nu}\left(\mathbf{r}^{\prime}\right)\right]\right]\right\rangle=n_{0}(\mathbf{r}) \partial_{\mu} \delta\left(\mathbf{r}-\mathbf{r}^{\prime}\right) \partial_{\nu} V_{0}(\mathbf{r})$.

(iii) For the interaction part, we need the two types of commutators

$$
\begin{aligned}
& m^{2}\left[j_{1 \mu}(\mathbf{r}),\left[V_{C}\left(\left|\mathbf{r}_{1}-\mathbf{r}_{2}\right|\right), j_{1 \nu}\left(\mathbf{r}^{\prime}\right)\right]\right] \\
& \quad=\delta\left(\mathbf{r}-\mathbf{r}_{1}\right) \partial_{\mu} \delta\left(\mathbf{r}-\mathbf{r}^{\prime}\right) \partial_{\nu}^{\prime} V_{C}\left(\left|\mathbf{r}^{\prime}-\mathbf{r}_{2}\right|\right), \\
& m^{2}\left[j_{1 \mu}(\mathbf{r}),\left[V_{C}\left(\left|\mathbf{r}_{1}-\mathbf{r}_{2}\right|\right), j_{2 \nu}\left(\mathbf{r}^{\prime}\right)\right]\right] \\
& \quad=\delta\left(\mathbf{r}-\mathbf{r}_{1}\right) \delta\left(\mathbf{r}^{\prime}-\mathbf{r}_{2}\right) \partial_{\mu} \partial_{\nu}^{\prime} V_{C}\left(\left|\mathbf{r}-\mathbf{r}^{\prime}\right|\right) .
\end{aligned}
$$

Then the interaction part can be rewritten in terms of the ground-state (equilibrium) pair correlation function $g\left(\mathbf{r}, \mathbf{r}^{\prime}\right)$ of Eq. (17) as

$$
\begin{aligned}
& m^{2}\left\langle\left[j_{\mu}(\mathbf{r}),\left[\frac{1}{2} \sum_{n \neq m} V_{C}\left(\left|\mathbf{r}_{n}-\mathbf{r}_{m}\right|\right), j_{\nu}\left(\mathbf{r}^{\prime}\right)\right]\right]\right\rangle=n_{0}(\mathbf{r}) \\
& \quad \times\left[\int\left[g\left(\mathbf{r}, \mathbf{r}^{\prime \prime}\right)-1\right] \partial_{\mu} \delta\left(\mathbf{r}-\mathbf{r}^{\prime}\right) \partial_{\nu}^{\prime} n_{0}\left(\mathbf{r}^{\prime \prime}\right) V_{C}\left(\left|\mathbf{r}^{\prime}-\mathbf{r}^{\prime \prime}\right|\right) d \mathbf{r}^{\prime \prime}\right. \\
& \quad+n_{0}\left(\mathbf{r}^{\prime}\right)\left[g\left(\mathbf{r}, \mathbf{r}^{\prime}\right)-1\right] \partial_{\mu} \partial_{\nu}^{\prime} V_{C}\left(\left|\mathbf{r}-\mathbf{r}^{\prime}\right|\right) \\
& \left.\quad+\partial_{\mu} \delta\left(\mathbf{r}-\mathbf{r}^{\prime}\right) \partial_{\nu}^{\prime} V_{H}\left(\mathbf{r}^{\prime}\right)+n_{0}\left(\mathbf{r}^{\prime}\right) \partial_{\mu} \partial_{\nu}^{\prime} V_{C}\left(\left|\mathbf{r}-\mathbf{r}^{\prime}\right|\right)\right], \quad(\mathrm{A} 3)
\end{aligned}
$$

where

$$
\left.V_{H}(\mathbf{r})=\int n_{0}\left(\mathbf{r}^{\prime}\right) V_{C}\left(\mid \mathbf{r}-\mathbf{r}^{\prime}\right) \mid\right) d \mathbf{r}^{\prime}
$$

is Hartree potential.

(iv) A considerable simplification comes from the force balance equation

$$
\begin{gathered}
\partial_{\nu} T_{\nu \mu}(\mathbf{r})+n_{0}(\mathbf{r}) \partial_{\mu} V_{0}(\mathbf{r})+n_{0}(\mathbf{r}) \int g\left(\mathbf{r}, \mathbf{r}^{\prime}\right) n_{0}\left(\mathbf{r}^{\prime}\right) \\
\times \partial_{\mu} V_{C}\left(\left|\mathbf{r}-\mathbf{r}^{\prime}\right|\right) d \mathbf{r}^{\prime}=0
\end{gathered}
$$

the latter immediately following from the obvious relation $\left\langle\left[j_{\mu}(\mathbf{r}), H_{0}\right]\right\rangle=0$.

(v) $M_{\mu \nu}\left(\mathbf{r}, \mathbf{r}^{\prime}\right)$ is obtained by combining Eqs. (A1)-(A4). Then $M_{\mu \nu}^{s}\left(\mathbf{r}, \mathbf{r}^{\prime}\right)$ is constructed from $M_{\mu \nu}\left(\mathbf{r}, \mathbf{r}^{\prime}\right)$ by replacing $V_{0}$ with $V_{s}=V_{0}+V_{H}+V_{\mathrm{xc}}$ and setting the interaction term to zero. We finally arrive at Eq. (13) by the use of Eq. (7).

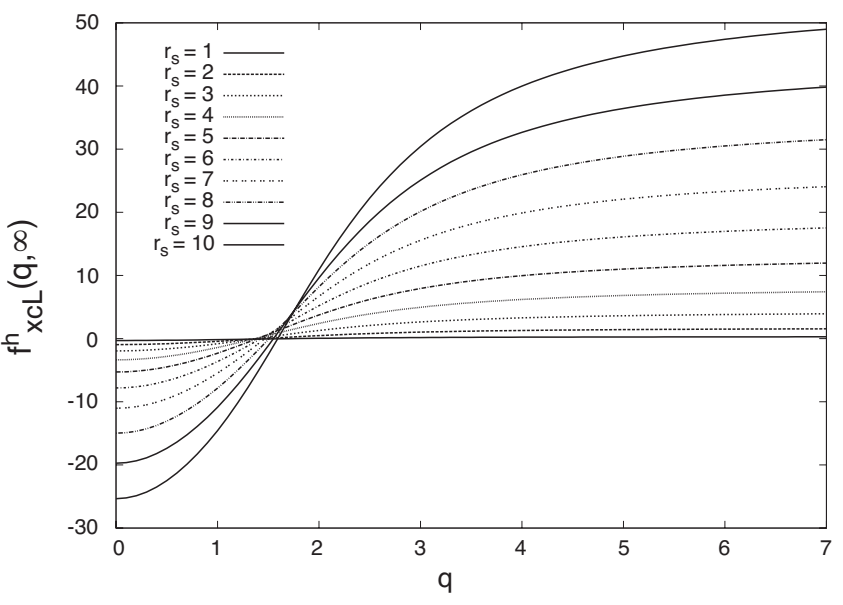

FIG. 1. High-frequency limit of the longitudinal xc kernel for the homogenous electron gas plotted as function of $q$ for different values of $r_{s}$. Asymptotically the only finite contribution is due to the term containing the correlation kinetic energy.

\section{APPENDIX B: THE ANTIADIABATIC LIMIT OF THE XC KERNEL FOR THE HOMOGENEOUS ELECTRON GAS}

The tensor xc kernel of a homogeneous electron gas has the form ${ }^{2}$

$$
\left[\hat{f}_{\mathrm{xc}}^{h}(q, \omega)\right]_{i j}=\frac{q^{2}}{\omega^{2}}\left[f_{\mathrm{xcL}}^{h}(q, \omega) \hat{q}_{i} \hat{q}_{j}+f_{\mathrm{xc} T}^{h}(q, \omega)\left(\delta_{i j}-\hat{q}_{i} \hat{q}_{j}\right)\right],
$$

The longitudinal (L) component of the kernel coincides with the scalar xc kernel. In the high-frequency limit, by using the $f$-sum rule and the third-moment sum rule for the density-density response functions (or the first-moment sum rule for the current-current response functions) for the interacting and noninteracting gas, ${ }^{22}$ one readily obtains

$$
f_{\mathrm{xcL}}^{h}(q, \infty)=f_{\mathrm{xcL}}^{(1)}(q, \infty)+f_{\mathrm{xcL}}^{(2)}(q, \infty)
$$

where

$$
f_{\mathrm{xcL}}^{(1)}(q, \infty)=\frac{4}{3} \alpha r_{s}^{2} \int_{0}^{\infty} d k\left[S\left(k, r_{s}\right)-1\right] f(k / q)
$$

and

$$
f_{\mathrm{xcL}}^{(2)}(q, \infty)=\frac{8}{3} \pi r_{s}^{3} t_{c}\left(r_{s}\right)
$$

with

$$
f(x)=\frac{x^{2}}{2}\left[\frac{5}{3}-x^{2}+\frac{\left(x^{2}-1\right)^{2}}{2 x} \ln \left(\left|\frac{1+1 / x}{1-1 / x}\right|\right)\right]
$$

$t_{c}\left(r_{s}\right)$ is the correlation kinetic energy and $S\left(k, r_{s}\right)$ is the static structure factor. We emphasize that the contribution due to the correlation kinetic energy, $f_{\mathrm{xcL}}^{(2)}(q, \infty)$, is independent of $q$. In the expressions above the atomic units in which $\hbar=e^{2}$ $=m=1$ are used and the wave vectors are expressed in units of the Fermi wave vector $q_{F}=\alpha / r_{s}$, with $\alpha=(9 \pi / 4)^{1 / 3}$.

In the same limit, the transverse component is given by 


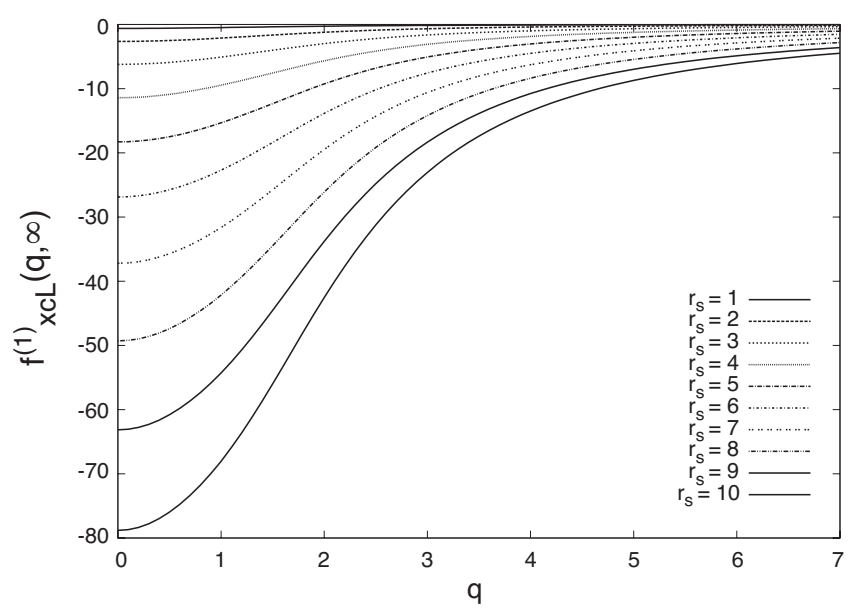

FIG. 2. Here the quantity in Fig. 1 is plotted by neglecting $f_{\mathrm{xcL}}^{(2)}(q, \infty)$ [Eq. (B4)].

$$
f_{\mathrm{xcT}}^{h}(q, \infty)=-\frac{1}{2} f_{\mathrm{xcL}}^{(1)}(q, \infty)+\frac{1}{3} f_{\mathrm{xcL}}^{(2)}(q, \infty) .
$$

For the calculation of $S\left(k, r_{s}\right)$ we have employed the analytic static structure factor provided in Ref. 26, and the correlation kinetic energy has been calculated from the parametrized correlation energy of Appendix B of the same reference, by making use of the virial theorem. The resulting quantities $f_{\mathrm{xcL}}^{h}(q, \infty), f_{\mathrm{xcL}}^{(1)}(q, \infty)$ and $f_{\mathrm{xcT}}^{h}(q, \infty)$ are plotted in Figs. 1-3, respectively. Among other facts, these plots show that the contribution due to the correlation kinetic energy is very important at any $q-$ a fact already pointed out by Iwamoto and Gross ${ }^{27}$ for the longitudinal component at $q=0$. Moreover, it is clear that $f_{\mathrm{xcL}}^{(2)}(q, \infty)$ can be identified as the $q \rightarrow \infty$ limit of $f_{\mathrm{xcL}}^{h}(q, \infty)$. Overall, $f_{\mathrm{xcL}(T)}^{h}(q, \infty)$ has a strong dependence on both $q$ and $r_{s}$. From Figs. 1 and 3, it is evi-

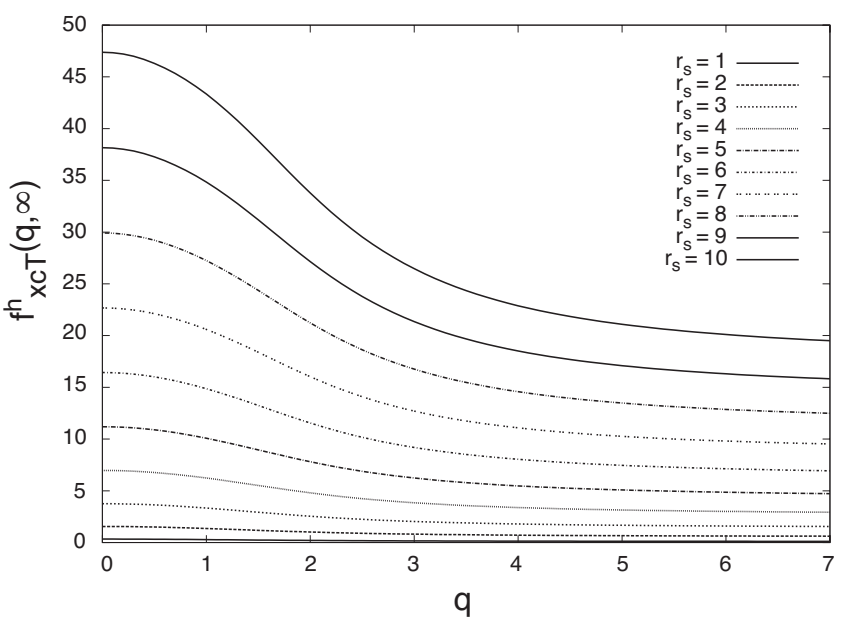

FIG. 3. High-frequency limit of the transverse xc kernel for the homogenous electron gas plotted as function of $q$ for different values of $r_{s}$. Asymptotically the only finite contribution is due to the term containing the correlation kinetic energy.

dent that $f_{\mathrm{xcL}}^{h}(q, \infty)$ and $f_{\mathrm{xcT}}^{h}(q, \infty)$ have very different ranges of values. We observe that while $f_{\mathrm{xcT} T}^{h}(q, \infty)$ is always positive, $f_{\mathrm{xcL}}^{h}(q, \infty)$ changes sign at about a fixed value of $q / k_{F}$ for all $r_{s}$ : this suggests that the $r_{s}$ dependence of $f_{\mathrm{xcL}}^{h}(q, \infty)$ with $q$ expressed in units of $k_{F}$ may be an overall scale factor. Clearly, the fact that $f_{\mathrm{xcL}(T)}^{h}(q, \infty)$ go to a constant in the large $q$ limit implies that the corresponding local field factors diverge as $q^{2}$. This is also observed at finite frequency, and, indeed all the way to zero frequency, as first noticed by Holas $^{28}$ and then confirmed by quantum Monte Carlo ${ }^{3}$ and diagrammatic calculations. ${ }^{29}$ In particular, the exact relation $f_{\mathrm{xcL}}^{h}(q \rightarrow \infty, \infty)=-3 f_{\mathrm{xcL}}^{h}(q \rightarrow \infty, 0)$ (Ref. 28) is well satisfied.
${ }^{1}$ E. K. U. Gross and W. Kohn, Phys. Rev. Lett. 55, 2850 (1985).

${ }^{2}$ G. Vignale and W. Kohn, Phys. Rev. Lett. 77, 2037 (1996).

${ }^{3}$ S. Moroni, D. M. Ceperley, and G. Senatore, Phys. Rev. Lett. 75, 689 (1995).

${ }^{4}$ M. Corradini, R. Del Sole, G. Onida, and M. Palummo, Phys. Rev. B 57, 14569 (1998).

${ }^{5}$ B. Dabrowski, Phys. Rev. B 34, 4989 (1986).

${ }^{6}$ Z. Qian and G. Vignale, Phys. Rev. B 65, 235121 (2002).

${ }^{7}$ K. Sturm and A. Gusarov, Phys. Rev. B 62, 16474 (2000).

${ }^{8}$ Y.-H. Kim and A. Görling, Phys. Rev. B 66, 035114 (2002).

${ }^{9}$ P. Ghosez, X. Gonze, and R. W. Godby, Phys. Rev. B 56, 12811 (1997).

${ }^{10}$ L. Reining, V. Olevano, A. Rubio, and G. Onida, Phys. Rev. Lett. 88, 066404 (2002).

${ }^{11}$ G. Adragna, R. Del Sole, and A. Marini, Phys. Rev. B 68, 165108 (2003).

${ }^{12}$ F. Sottile, V. Olevano, and L. Reining, Phys. Rev. Lett. 91, 056402 (2003).

${ }^{13}$ R. Stubner, I. V. Tokatly, and O. Pankratov, Phys. Rev. B 70, 245119 (2004).

${ }^{14}$ V. U. Nazarov, J. M. Pitarke, Y. Takada, G. Vignale, and Y.-C.
Chang, Phys. Rev. B 76, 205103 (2007).

${ }^{15}$ V. U. Nazarov, J. M. Pitarke, Y. Takada, G. Vignale, and Y.-C. Chang, Int. J. Mod. Phys. B 22, 3813 (2008).

${ }^{16}$ V. U. Nazarov, G. Vignale, and Y.-C. Chang (unpublished).

${ }^{17}$ V. U. Nazarov, G. Vignale, and Y.-C. Chang, Phys. Rev. Lett. 102, 113001 (2009).

${ }^{18}$ J. Tao, X. Gao, G. Vignale, and I. V. Tokatly, Phys. Rev. Lett. 103, 086401 (2009).

${ }^{19}$ X. Gao, J. Tao, G. Vignale, and I. V. Tokatly, Phys. Rev. B 81, 195106 (2010).

${ }^{20}$ I. V. Tokatly, Phys. Rev. B 75, 125105 (2007).

${ }^{21}$ I. V. Tokatly, Phys. Chem. Chem. Phys. 11, 4621 (2009).

${ }^{22} \mathrm{G}$. F. Giuliani and G. Vignale, Quantum Theory of the Electron Liquid (Cambridge University Press, Cambridge, 2005).

${ }^{23} \mathrm{We}$ focus on the zero-temperature case for simplicity but it is evident that everything we say can be easily generalized to an equilibrium ensemble of states at finite temperature.

${ }^{24}$ L. D. Landau and E. M. Lifshitz, Theory of Elasticity, Course of theoretical physics Vol. 7, 3rd ed. (Butterworth-Heinemann, London, 1986)

${ }^{25}$ R. Del Sole, G. Adragna, V. Olevano, and L. Reining, Phys. Rev. 
B 67, 045207 (2003).

${ }^{26}$ P. Gori-Giorgi, F. Sacchetti, and G. B. Bachelet, Phys. Rev. B 61, 7353 (2000).

${ }^{27}$ N. Iwamoto and E. K. U. Gross, Phys. Rev. B 35, 3003 (1987).
${ }^{28}$ A. Holas, in Strongly Coupled Plasma Physics, NATO Advanced Study Institute Series B: Physics, edited by F. J. Rogers and H. Dewitt (Plenum Press, New York, 1986), Vol. 154, p. 463.

${ }^{29}$ G. Vignale, Phys. Rev. B 38, 6445 (1988). 